\title{
Altered pulmonary artery endothelial-smooth muscle cell interactions in experimental congenital diaphragmatic hernia
}

\author{
Shannon N. Acker ${ }^{1,2}$, Gregory J. Seedorf2,3, Steven H. Abman ${ }^{2,3}$, Eva Nozik-Grayck ${ }^{3}$, Katherine Kuhn ${ }^{2}$, David A. Partrick and \\ Jason Gien ${ }^{2,3}$
}

BACKGROUND: Pulmonary hypertension (PH) secondary to vascular remodeling contributes to poor outcomes in congenital diaphragmatic hernia (CDH), however mechanisms responsible are unknown. We hypothesized that pulmonary artery endothelial cell (PAEC) dysfunction contributes to smooth muscle cell (SMC) hyperplasia in experimental $\mathrm{CDH}$.

METHODS: PAEC and SMC were isolated from fetal sheep with experimental $\mathrm{CDH}$ and controls. SMC growth was assessed alone and with SOD plus catalase and during coculture with control or CDH PAEC with and without ET-1 siRNA transfection. ET-1 protein was measured in PAEC and SMC lysates and supernatant. ROS production was measured in normal and $\mathrm{CDH}$ PAECs with and without ET-1 siRNA. PAEC growth and tube formation were measured with SOD plus catalase.

RESULTS: CDH SMC growth was decreased and increased with coculture with CDH PAEC more than control PAEC. Treatment of CDH PAEC with SOD plus catalase or ET-1 siRNA prevented the increase in SMC growth seen with coculture. ET-1 protein was increased in CDH PAEC and SMC. ROS production was increased in CDH PAEC and decreased with ET-1 SiRNA. SOD plus catalase restored CDH PAEC growth and tube formation. CONCLUSION: PAEC dysfunction in experimental $\mathrm{CDH}$ increases SMC proliferation via ET-1 induced ROS production by PAEC.

C ongenital diaphragmatic hernia $(\mathrm{CDH})$ is characterized by pulmonary hypoplasia and abnormal development of the pulmonary vasculature in utero, including impaired angiogenesis and hypertensive vascular remodeling $(1,2)$. The severity of pulmonary hypoplasia and degree of pulmonary hypertension determine outcomes in infants with $\mathrm{CDH}(3-6)$. However, prenatal factors contributing to the development of vascular remodeling and impaired angiogenesis in utero, remain unclear. Pulmonary artery endothelial cell (PAEC) dysfunction contributes to the development of pulmonary hypertension in experimental $\mathrm{CDH}(7,8)$. We have previously reported that PAECs harvested from an experimental model of $\mathrm{CDH}$ demonstrate impaired growth and tube formation in vitro and decreased extracellular superoxide dismutase protein expression (7). While PAEC dysfunction contributes to impaired angiogenesis in $\mathrm{CDH}$, little is known about mechanisms responsible for vascular remodeling in utero. To date, there are no reports describing the effect of altered PAEC signaling on pulmonary artery smooth muscle cell (PASMC) growth in $\mathrm{CDH}$.

Previously published reports that focus on PAEC:PASMC interactions have demonstrated that endothelial cells induce recruitment, proliferation, and differentiation of SMC via production of growth factors (9-14). These data suggest that PAEC, specifically hypoxic PAEC, may contribute to PASMC proliferation by inducing a transition from a contractile phenotype to a proliferative phenotype via production of growth factors. Whether changes in PASMC phenotype or abnormal PAEC signaling are responsible for PASMC hyperplasia and vascular remodeling in experimental $\mathrm{CDH}$ has not been previously evaluated.

Altered PAEC signaling contributes to the development of pulmonary hypertension with decreased production of vasodilators, such as nitric oxide (NO), and increased release of vasoconstrictors, such as endothelin-1 (ET-1), both of which are derived from the endothelium (15-17). ET-1 acts as both a vasoconstrictor and mitogen for PASMC (18); possibly via production of reactive oxygen species (ROS), including superoxide and hydrogen peroxide, both of which can cause vasoconstriction and PASMC growth $(15,19)$. ET-1 levels are elevated in the serum of infants with $\mathrm{CDH}$ and persistent pulmonary hypertension of newborn (PPHN), with higher ET-1 levels correlating with more severe pulmonary hypertension and mortality (20-22). Previous work in animal models of $\mathrm{CDH}$ have demonstrated that ET-1 levels are increased in lung tissue (23) and therapeutic strategies that decrease ET-1 signaling lead to improvements in vascular remodeling (24). These studies in diverse models of pulmonary hypertension (PH) confirm that ET-1 is an important mediator of $\mathrm{PH}$, but the mechanisms by which ET- 1 contributes to vascular remodeling are unclear. The potential contribution of ET- 1 to the regulation of PASMC growth has not been studied and whether altered PAEC signaling affects PASMC growth and contributes to lung vascular remodeling in $\mathrm{CDH}$ is unknown.

Prior studies in experimental animal models of pulmonary hypertension and lung injury have demonstrated that a deficit of extracellular superoxide dismutase (SOD) impairs reactive oxygen species (ROS) scavenging and contributes to the

'Department of Surgery, University of Colorado School of Medicine, Aurora, Colorado; ${ }^{2}$ Department of Pediatrics, The Pediatric Heart Lung Center, Children's Hospital Colorado, University of Colorado School of Medicine, Aurora, Colorado; ${ }^{3}$ Department of Pediatrics, University of Colorado School of Medicine, Aurora, Colorado. Correspondence: Shannon N. Acker (shannon.acker@ucdenver.edu)

Received 29 May 2014; accepted 19 October 2014; advance online publication 4 February 2015. doi:10.1038/pr.2015.13 
a

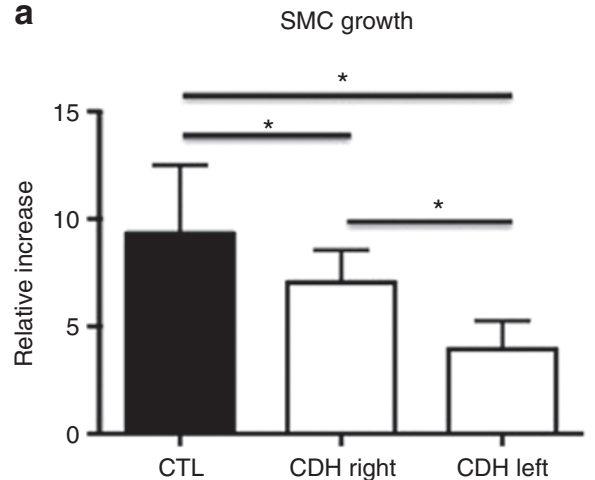

b

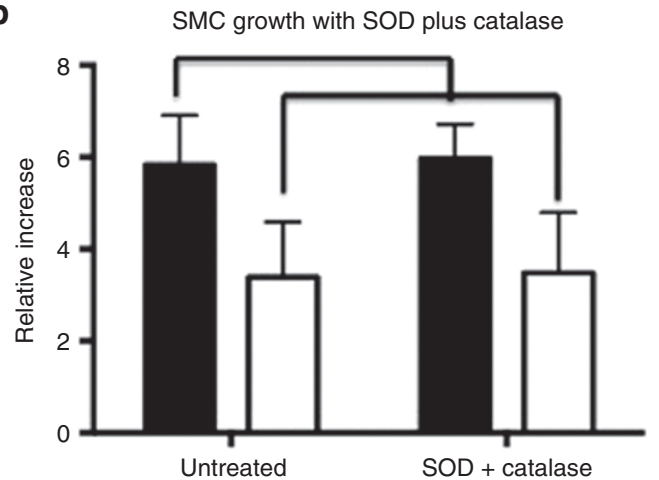

C

SMC growth with bosentan

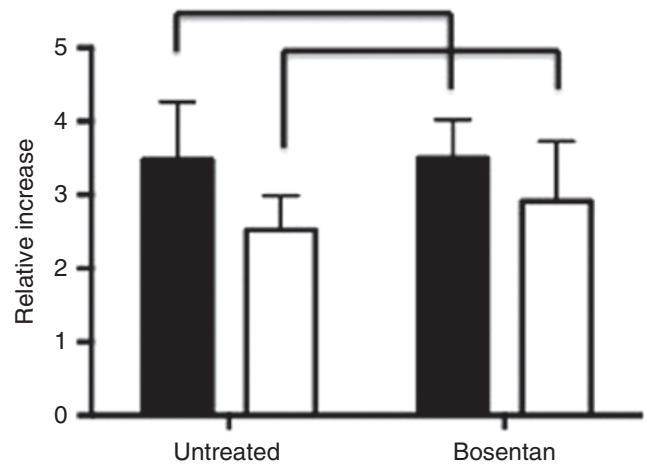

Figure 1. PASMC growth in experimental $C D H$. In comparison with cells from control animals, PASMC from right CDH animals grew significantly slower. (a) PASMC from left grew slower than those from the right in CDH animals. The addition of SOD plus catalase had no effect on the growth of PASMC from either control or right CDH PASMC (b; $P$ not significant for all comparisons noted). Bars represent standard error from the mean. Bosentan treatment had no effect on PASMC growth from either control or right CDH PASMC (c; $P$ not significant for all comparisons noted). ${ }^{*} P<0.05$.

development of pulmonary fibrosis and vascular remodeling (25). In this setting, exogenous SOD administration reduces inflammatory changes, pulmonary vascular resistance, and lung injury (25-27). We have previously demonstrated decreased SOD protein expression in PAEC from fetal sheep with experimental CDH (7). However, whether decreased PAEC SOD protein expression alters PASMC function remains unknown.

Based on these data, we hypothesized that PAEC dysfunction with increased ET-1 and ROS production directly alters PASMC function and contributes to pulmonary vascular remodeling in $\mathrm{CDH}$. To test this hypothesis, we performed a series of in vitro experiments with PAEC and PASMC isolated from fetal sheep with $\mathrm{CDH}$ and age matched controls to determine mechanisms responsible for vascular remodeling in utero. We report that increased ET-1 protein expression and ROS production by PAEC from $\mathrm{CDH}$ fetal sheep directly alters PAEC:PASMC interactions and contributes to CDH PASMC proliferation. ET-1 knockdown with siRNA or exogenous SOD plus catalase restores PAEC:PASMC interactions to normal.

\section{RESULTS}

PASMC Growth is Decreased in CDH Lambs, but Not Affected by the Addition of SOD Plus Catalase or Bosentan

In comparison to control PASMCs, CDH PASMC growth is decreased by $24 \%$ on the right and $58 \%$ on the left $(P<0.05$;
Figure 1a). The addition of SOD plus catalase did not affect the growth of PASMC from either control or right $\mathrm{CDH}$ pulmonary arteries (Figure 1b). Bosentan had no effect on the growth of PASMC from control or right $\mathrm{CDH}$ pulmonary arteries (Figure 1c).

In Coculture Experiments, PAECs Isolated From Fetal Sheep With Experimental CDH Have a Greater Effect on PASMC Growth Than Control PAEC

PASMC from control or CDH animals were placed in coculture with PAEC from either control or $\mathrm{CDH}$ animals for 4 d. Coculture of CDH PAEC with control PASMC stimulated PASMC to grow 35\% faster than when cocultured with control PAEC $(P<0.01$, Figure 2$)$. Coculture of CDH PASMC with PAEC from either control or $\mathrm{CDH}$ animals, demonstrated that PASMC cultured with CDH PAEC grew 54\% faster than PASMC cultured with control PAEC $(P<0.01$, Figure 2$)$.

ET-1 Protein is Increased in Whole Cell Lysates and Supernatant From CDH PAEC and PASMC, but ET-A and ET-B Receptor Levels are Unchanged

Western blot analysis of PAEC and PASMC whole cell lysates from normal and $\mathrm{CDH}$ lambs demonstrated increased ET-1 protein in CDH PAEC and PASMC (Figure 3). When compared to controls, CDH PAEC ET-1 protein expression was increased by $31 \%(P \leq 0.01)$ (Figure $3 a$ ) and CDH PASMC 
ET-1 protein expression was increased by $44 \%(P<0.01)$ (Figure $3 b$ ). ET-1 protein was also increased in the supernatant from $\mathrm{CDH}$ PAEC and PASMC. When compared to controls, ET-1 protein in the supernatant from CDH PAEC

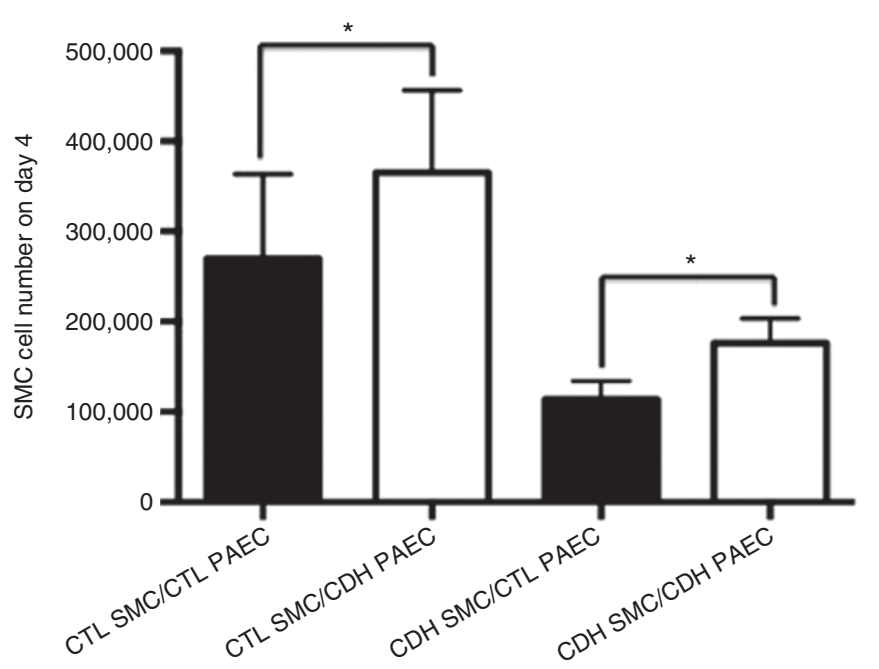

Figure 2. Effect of PAEC on PASMC growth in coculture conditions. Coculture of CDH PAEC with PASMC increases control and CDH PASMC growth more than measured during coculture with control PAEC. Bars represent standard error from the mean. ${ }^{*} P<0.05$.

a

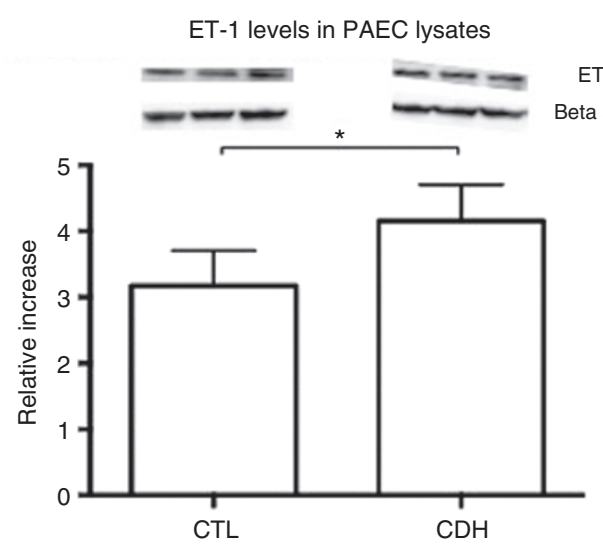

C

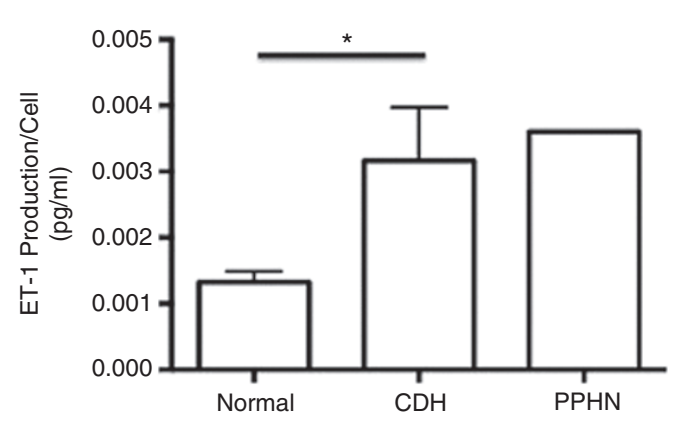

(Figure 3c) and PASMC (Figure 3d) was increased by $139 \%$ $(P<0.01)$. No difference in the levels of ET-A or ET-B receptor protein expression between control and CDH PASMC were seen $(P>0.2$ for all comparisons, data not shown).

\section{SOD Plus Catalase Restores Growth and Tube Formation in $\mathrm{CDH}$ PAEC}

At baseline, the growth of CDH PAEC is decreased by $38 \%$ in comparison with controls $(P=0.02$, Figure $4 a)$. The addition of SOD plus catalase restored growth of CDH PAEC to that of nonstimulated control PAEC ( $P=0.11$, Figure 4a). The ability of PAEC to spontaneously form vascular networks was also decreased in CDH PAEC (Figure 4b). When compared to PAEC from control animals, the number of branch points per high power field (HPF) was decreased by $18 \%(P<0.0001)$. The addition of SOD plus catalase stimulated tube formation in both control and CDH PAEC by 14 and 20\% respectively $(P<0.0001$ for both), restoring the ability of CDH PAEC to form tubular structures to rates comparable to nonstimulated control PAEC $(P=\mathrm{NS})$.

\section{ROS Production is Increased in CDH PAEC and SMC}

In comparison to controls, PAEC from $\mathrm{CDH}$ lambs produced $186 \%$ more ROS $(P<0.001$, Figure 5a). SOD plus catalase was used as a negative control and completely eliminated the ROS

b

ET-1 levels in PASMC lysates

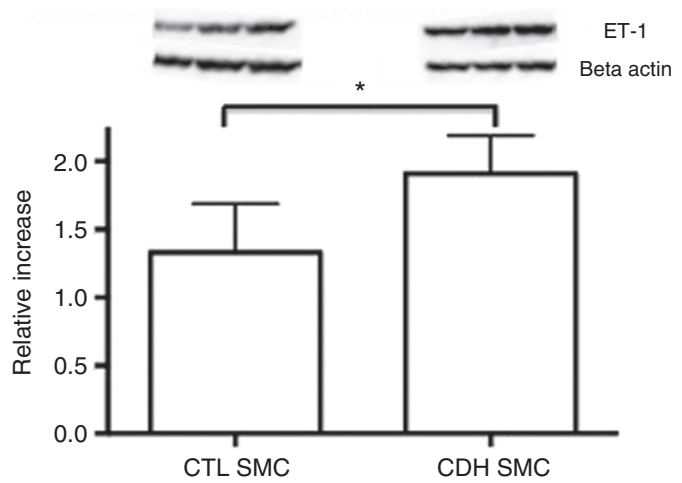

d

ET-1 levels in media from PASMC

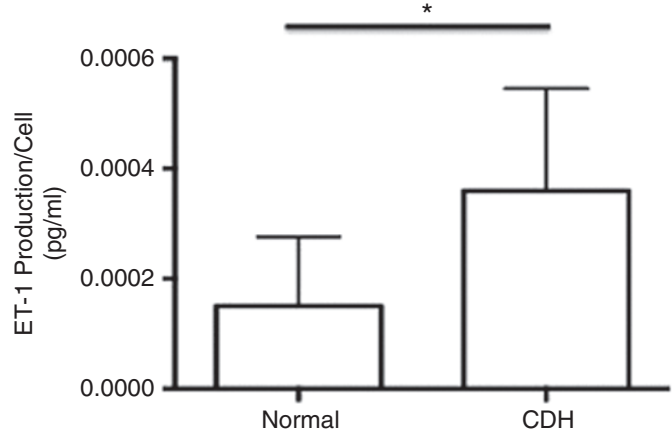

Figure 3. Measurement of ET-1 protein levels in cellular lysates and cell culture media. ET-1 protein levels were increased in (a) CDH PAEC and (b) PASMC whole cell lysates when compared to controls. Bars represent standard error from the mean. ET-1 protein levels in the supernatant were increased by $139 \%(P<0.01)$ from (c) CDH PAEC and (d) CDH PASMC when compared to media from control cells. PPHN PASMC were used as a control as PPHN PASMC are known to produce increased levels of ET-1 compared to control PASMC. PPHN, persistent pulmonary hypertension of newborn. 
a

Effect of SOD + catalase on PAEC growth

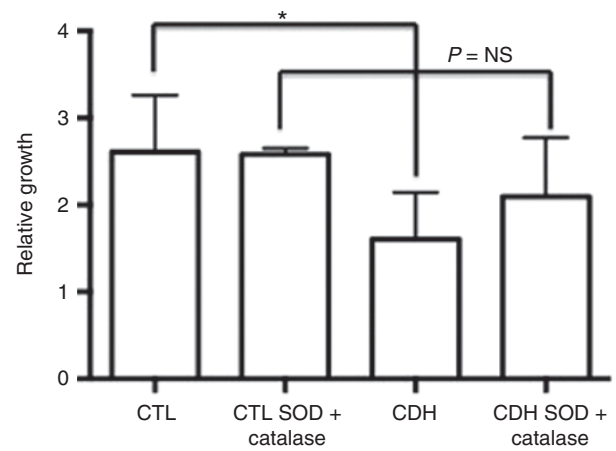

b

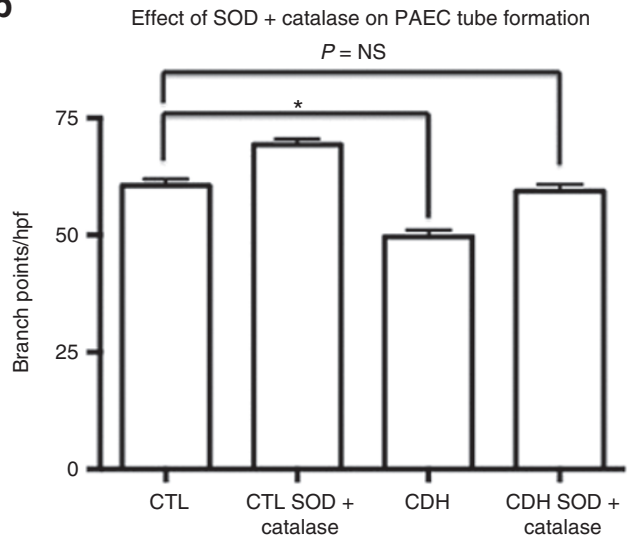

Figure 4. SOD and catalase restores CDH PAEC growth and tube formation. (a) CDH PAEC growth was decreased by $38 \%$ in comparison with control PAEC. (b) The addition of SOD plus catalase restores growth of CDH PAEC. Tube formation is reduced in CDH PAEC by $18 \%$ when compared to controls. The addition of SOD plus catalase restores tube structure formation of CDH PAEC to levels of nonstimulated control PAEC. Bars represent standard error from the mean. hpf, high power field. ${ }^{*} P<0.05$.

a

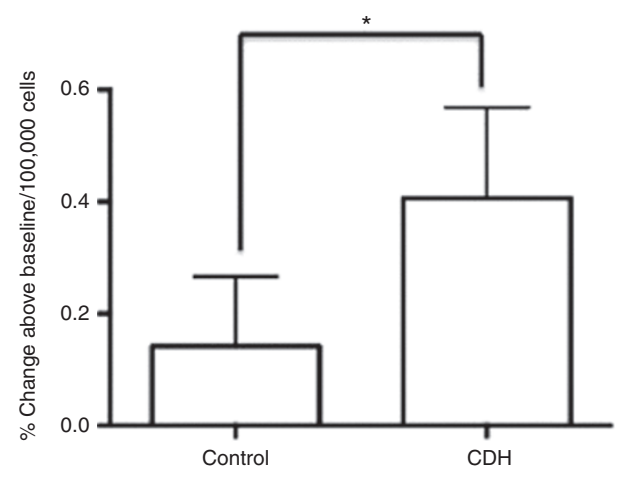

b

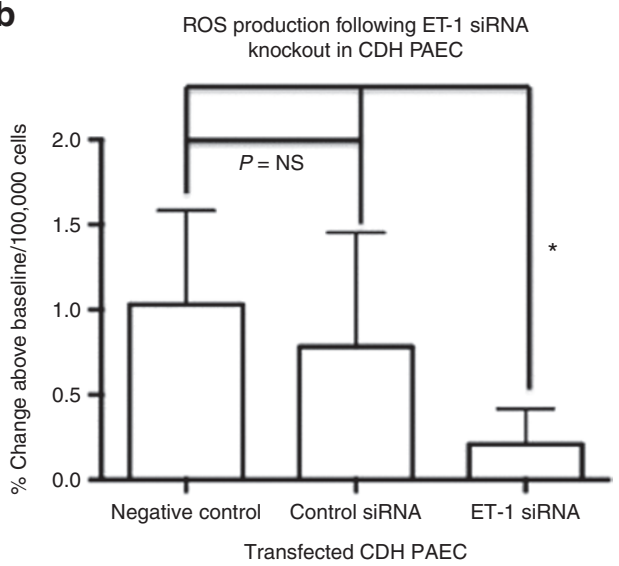

Figure 5. ROS production in PAEC. (a) At baseline, ROS production is increased in CDH PAEC. (b) Transfection of CDH PAEC with ET-1 siRNA decreased ROS production to baseline levels. Transfection with either a negative control or control siRNA did not decrease ROS production. Bars represent standard error from the mean. ${ }^{*} P<0.05$.

signal in both control and CDH PAEC (data not shown). SMC from CDH lambs produced $18 \%$ more ROS than SMC from control animals (data not shown).

\section{ET-1 siRNA Decreases ROS Production in CDH PAEC and SMC} ET-1 silencing with siRNA in CDH PAEC eliminated ROS production (levels did not differ from baseline fluorescence of $\mathrm{CDH}$ cells without the addition of the CellROX agent) (Figure 5b). The addition of transfection media alone (transfection negative control) or control siRNA (scramble) did not decrease ROS production in CDH PAEC (Figure 5b). The same pattern was demonstrated when the experiment was performed with CDH SMC (data not shown).

\section{SOD Plus Catalase Prevents CDH PAEC From Increasing PASMC} Growth Under Coculture Conditions

PASMC from control animals were cocultured with PAEC from both control and $\mathrm{CDH}$ animals with and without the addition of SOD plus catalase. The addition of SOD plus catalase to the coculture set up prevented CDH PAEC from increasing PASMC growth above levels seen when PASMC were cocultured with control PAEC. Adding SOD plus catalase to CDH PAEC coculture set up lead to PASMC growth rates that were identical to PASMC growth when cocultured with control PAEC (Figure 6a).

\section{ET-1 siRNA Prevents CDH PAEC From Increasing PASMC Growth Under Coculture Conditions}

PASMC from control animals were cocultured with PAEC from control and $\mathrm{CDH}$ animals after transfection with ET-1 siRNA, scramble siRNA or a negative control. CDH PAEC transfected with either a negative control (transfection media only) or scramble siRNA caused PASMC to grow 36\% faster than PASMC cocultured with control PAEC transfected in the same fashion $(P<0.0001$, Figure $6 b)$. Transfection of CDH PAEC with ET-1 siRNA prior to coculture with control PASMC decreased the rate of PASMC growth to similar values measured during coculture of PASMC with nontransfected control PAEC (Figure 6b). 
a

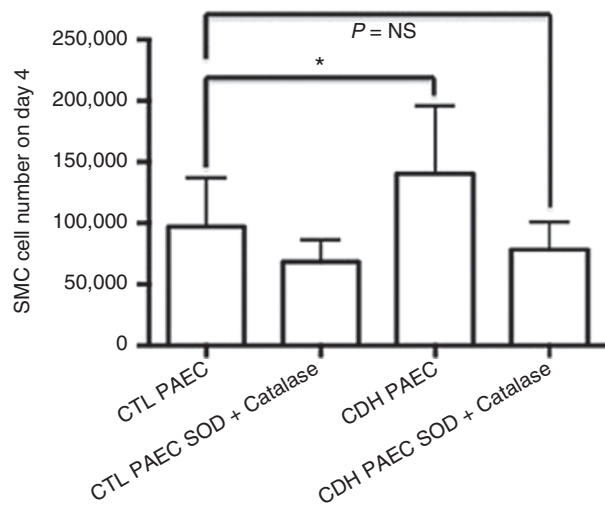

b

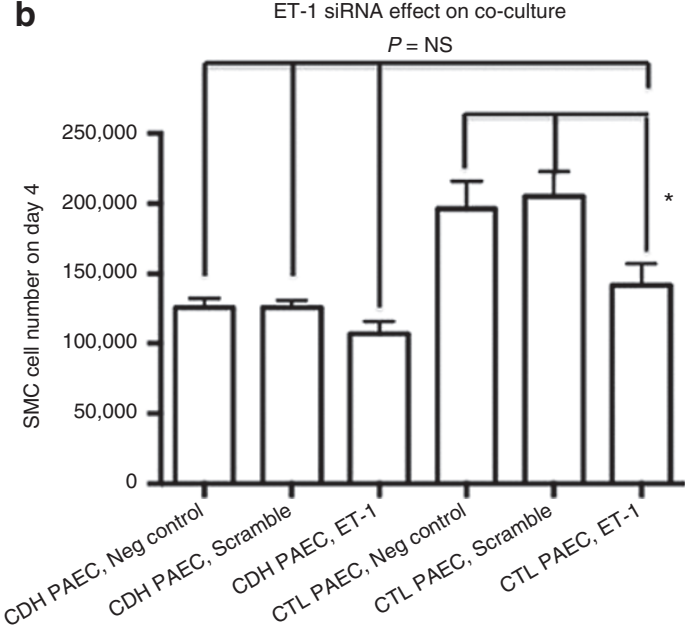

Figure 6. Role of ROS on PAEC-PASMC signaling during coculture. (a) SOD plus catalase treatment prevented CDH PAEC from stimulating control PASMC to grow faster than during coculture with control PAEC. (b) Transfection of CDH PAEC with ET-1 siRNA also prevented CDH PAEC from stimulating control PASMC growth. Bars represent standard error from the mean. ${ }^{*} P<0.05$.

\section{DISCUSSION}

We found that PAEC dysfunction is directly responsible for an altered PASMC phenotype in an experimental model of $\mathrm{CDH}$. PAEC dysfunction in $\mathrm{CDH}$ is characterized by elevated ROS production, which occurs secondary to increased ET-1 protein expression and production. Altered CDH PAEC function is restored to that of control PAEC following knockdown of ET-1 signaling or with exogenous SOD plus catalase treatment. In addition, PASMC growth is decreased in experimental $\mathrm{CDH}$ but is increased during coculture with $\mathrm{CDH}$ but not control PAEC. Knockdown of ET-1, or the addition of exogenous SOD with catalase inhibits the ability of $\mathrm{CDH}$ PAEC to stimulate PASMC growth. These findings demonstrate that PAEC dysfunction with increased ET-1 and ROS production contribute to increased PASMC proliferation and PASMC hyperplasia in $\mathrm{CDH}$.

These findings directly link PAEC dysfunction and PASMC hyperplasia in $\mathrm{CDH}$ through increased ET-1 induced production of ROS by PAEC. In vivo, endothelial and smooth muscle cells are in close proximity in the vessel wall; endothelialderived products play a key role in the regulation of PASMC function in normal and disease settings (9-14). Our findings demonstrate that PASMC hyperplasia in $\mathrm{CDH}$ is due to PAEC dysfunction and altered PAEC:PASMC interactions rather than a hyperproliferative PASMC phenotype. These findings differ significantly from other models of pulmonary hypertension. In experimental PPHN induced by ligation of the ductus arteriosus in utero, PASMC demonstrate significantly increased proliferation in vitro (28). Hyperplastic PASMC have also been found in rat models of $\mathrm{PH}$ and in human tissues from adult patients with idiopathic and heritable pulmonary arterial hypertension (29-31). In contrast to these reports, our findings demonstrate that experimental $\mathrm{CDH}$ in fetal sheep induces a PASMC phenotype that is distinct from non-CDH PPHN. While PASMC in non-CDH PPHN are hyperproliferative (28-31), PASMC from the CDH fetal sheep model are hypoproliferative. However, when grown in coculture with PAEC, altered CDH PAEC signaling leads to PASMC hyperproliferation, suggesting that the vascular remodeling that characterizes $\mathrm{CDH}$ results from altered PAEC signaling and not from independent PASMC proliferation. This is the first description of PAEC:PASMC interaction in vitro in experimental $\mathrm{CDH}$, and provides novel insights into mechanisms responsible for pulmonary vascular dysfunction in $\mathrm{CDH}$.

In addition to its role in the regulation of vascular tone, ET-1 is a potent PASMC mitogen (18). There are many reports from both human and animal studies describing elevated serum ET-1 levels in infants with $\mathrm{CDH}$ with a direct correlation between serum levels and disease severity $(20,21,32)$. ET-1 increases PASMC proliferation through direct mitogenic effects on PASMC (18) and increased ROS production (15). Our study confirms a link between ET-1 and ROS production in $\mathrm{CDH}$ PAEC. CDH PAEC ET-1 production and protein expression is increased and mediates increased ROS production verified by the finding that silencing of ET-1 expression with ET-1 siRNA decreased ROS production. While ET-1 production and protein expression was increased in CDH PASMC, ETA and ETB receptor expression was not different, confirming that changes in ET-1 production and expression are responsible for PAEC dysfunction and changes in PAEC:PASMC interactions, not altered receptor levels. This finding provides a novel mechanism for PASMC hyperplasia in $\mathrm{CDH}$ and further enhances our understanding of the contribution increased ET-1 plays to the development of vascular remodeling in $\mathrm{CDH}$. Utilizing a nitrofen induced rodent model of $\mathrm{CDH}$, previous groups have demonstrated that inhibition of ET-1 signaling leads to improved lung growth and prevents vascular remodeling (24). Our data provide further evidence identifying ET-1 signaling as a possible therapeutic target in $\mathrm{CDH}$.

Recently, we reported that SOD protein expression is decreased in PAECs isolated from fetal sheep with experimental $\mathrm{CDH}$ (7). This finding provided indirect evidence of 
increased ROS present in the $\mathrm{CDH}$ endothelium and a role for oxidative stress in contributing to PAEC dysfunction. Following up on this observation in our previous work, we now demonstrate increased ROS production in CDH PAEC, confirming the importance of oxidative stress in this model and suggesting an important role for antioxidant therapy. SOD catalyzes the conversion of superoxide to hydrogen peroxide with catalase then converting hydrogen peroxide to water. In our studies, both enzymes were required to restore PAEC function suggesting that both superoxide and hydrogen peroxide are responsible for PAEC dysfunction. Our findings are similar to previous work in a fetal sheep model of pulmonary hypertension, describing decreased levels of SOD with an associated increase in superoxide anions (33). Brennan et al. also demonstrated that SOD levels in the fetal lung were four times lower than in the adult lung suggesting that the fetal lung may be particularly sensitive to oxidative stress (33) making the potential impact of exogenous SOD therapy in the neonatal lung even more significant. Previous work has demonstrated the importance of ROS production to SMC proliferation, demonstrating that suppression of ROS production inhibits SMC proliferation and promotes apoptosis $(34,35)$. These studies are consistent with our findings and suggest ROS as key to the development of vascular remodeling in experimental models of pulmonary hypertension $(26,27)$.

Potential limitations of this study include the fact that there are a variety of PASMC mitogens and cell-signaling pathways responsible for regulating vascular remodeling; however, we have chosen only to focus on the role of ET-1, ROS, and SOD. While we have described a single potential target, there are a variety of other pathways that have not been explored, including the relationship between ROS and endothelial nitric oxide synthase (eNOS) in this $\mathrm{CDH}$ model. Furthermore, we have not yet fully characterized the definitive source of ROS production from the CDH PAEC and future work will aim to examine the source of ROS. While these data offer a potential therapeutic target, the data presented here are derived from in vitro studies only. Of note, significant differences were seen in the growth of the right and left PASMC from CDH animals and while it remains beyond the scope of the current work, future studies will be designed to help elucidate the mechanisms responsible for the differences in phenotype demonstrated on initial in vitro studies of PASMC from the right and left lungs of $\mathrm{CDH}$ animals. The cells used for the current experiments are derived from the conduit arteries, not the microvasculature. Using currently available techniques, we have been able to successfully isolated PAEC from the microvasculature of control animals. However, using identical techniques, we are unable to isolate PAEC from the microvasculature of the $\mathrm{CDH}$ animals. We acknowledge that pulmonary vascular development originates in the microvasculature; however, the strength of the data presented here is the use of organ specific cells from a developmentally relevant model of $\mathrm{CDH}$ and the use of multiple clones of cells from different animals. Future work will aim to clarify these interactions in the microvasculature as well.
In conclusion, we found that dysfunctional PAEC signaling contributes to PASMC hyperplasia and pulmonary hypertension in an experimental model of CDH. PAEC from CDH animals produce increased levels of ET-1 leading to increased ROS production. This, combined with decreased levels of SOD, an ROS scavenger, contribute to PASMC hyperplasia. Exogenous SOD or silencing of ET-1 signaling prevents the increase in PASMC growth secondary to altered PAEC signaling. We speculate that future therapies, which aim to supplement SOD or decrease ET-1 may restore altered PAEC signaling and PASMC growth, leading to improved patient outcomes in $\mathrm{CDH}$.

\section{METHODS}

\section{Experimental Model of $\mathrm{CDH}$}

Pregnant, mixed breed (Colombia-Rambouillet) ewes were used in this study. All procedures and protocols were reviewed and approved by the Animal Care and Use Committee of the University of Colorado Anschutz Medical Campus and followed the Guide for the Care and Use of Laboratory Animals established by the National Research Council. Surgery to induce a diaphragmatic defect was performed at $60-70 \mathrm{~d}$ gestation (full term $=147 \mathrm{~d}$ ) as previously described (7). Under sterile conditions, the uterus was externalized through a midline laparotomy incision and a small hysterotomy was performed to expose the fetus for a left sided thoracotomy. A defect in the left diaphragm of the fetus was surgically created and abdominal contents were gently pulled into the chest. The thoracotomy and uterine incisions were closed in sequence. The uterus was replaced inside the ewe and the laparotomy was closed. Animals were euthanized at $135 \mathrm{~d}$ gestation to harvest fetal lung tissues.

\section{Isolation and Culture of Fetal Ovine PAEC}

Left and right pulmonary arteries were isolated from fetal sheep with $\mathrm{CDH}$ and age-matched control animals ( $N=7 \mathrm{CDH}, 8$ controls). Proximal PAEC and PASMC were isolated as previously described $(36,37)$. Briefly, conduit pulmonary arteries were separated from fetal sheep and branching vessels were ligated. Collagenase was used to separate endothelial cells from the vessel wall. PAEC were plated and grown in Dulbecco's modified Eagle medium (DMEM) and 10\% fetal bovine serum (FBS). Endothelial cell phenotype was confirmed by a typical cobblestone appearance and positive immunostaining for von Willebrands Factor (vWF), eNOS, vascular endothelial (VE)cadherin, VEGF-R2 (KDR), positive uptake of ac-LDL and negative staining for desmin. PAEC from passages 3-7 were used for these experiments. Cells from left and right lung and from each animal were kept separate throughout all experiments. Initial experiments revealed no differences between PAEC obtained from the right and left lungs of the $\mathrm{CDH}$ animals with regard to growth or tube formation. For this reason, all data presented are compiled data from assays performed with PAEC from both left and right lungs of $\mathrm{CDH}$ animals. All cell culture experiments were performed in room air conditions (fraction of inspired oxygen 21\%).

\section{Isolation and Culture of Fetal Ovine PASMC}

Following digestion of the fetal conduit arteries for isolation of PAEC, the remaining vessel was used to isolate PASMC as previously described (28). PASMC identity was confirmed by characteristic morphology as well as positive immunostaining for $\alpha$-smooth muscle actin, calponin, caldesmon, and desmin with greater than $95 \%$ positivity. All experiments were conducted with cells at passages 3-7. Initial studies revealed that PASMC from the left CDH lung grew extremely slowly, limiting the ability to perform in vitro studies. For this reason, all data presented are based on studies performed with PASMC isolated from the right $\mathrm{CDH}$ lung, unless otherwise noted. For control experiments, cells were harvested from sheep that underwent fetal surgery and were exposed to the similar effects of maternal anesthesia as the CDH sheep. All experiments were performed in duplicate to ensure reproducibility. PAEC from eight control and 
seven $\mathrm{CDH}$ animals and PASMC from five control and four $\mathrm{CDH}$ animals were utilized for the experiments listed below.

\section{SMC Growth}

Fetal PASMC from normal and CDH lambs were plated on a six-well tissue culture plate at $1 \times 10^{6}$ cells/well in DMEM with $10 \% \mathrm{FBS}$ and allowed to adhere overnight. On day 0 , media was changed to DMEM with $2.5 \%$ FBS (as this was the lowest concentration that supported growth). On day 4 cells were removed from the wells using $0.25 \%$ trypsin/0.53 mmol/l ethylene-diaminetetraacetic acid (EDTA) digestion and counted using a hemocytometer. Absolute cell number on day 4 was compared to cell number at day 0 to determine the relative increase over time. Comparisons were made between control and $\mathrm{CDH}$ cells. This assay was repeated twice in the presence or absence of SOD (250 U/ml; Sigma Aldrich, St Louis, MO) plus catalase (26.3 U/ $\mathrm{ml}$; Worthington Biochemical, Lakewood, NJ) and Bosentan (a nonselective ET A and B receptor inhibitor) (1 mmol/l). Preliminary experiments evaluating the effects of SOD or catalase alone were performed, which revealed that either alone had no effect on either PAEC or PASMC growth (data not shown). For this reason, all experiments were performed with SOD plus catalase in combination.

\section{Coculture of PAEC With PASMC}

The effect of PAEC signaling on PASMC growth was assessed utilizing a coculture environment with Transwell cell culture plates (Corning, Corning, NY). $5 \times 10^{5}$ PASMC were plated on the bottom of a six well tissue culture plate in DMEM plus 10\% FBS and allowed to adhere overnight. In a separate six-well tissue culture plate, $1 \times 10^{6}$ PAEC were plated inside the Transwell in DMEM plus 10\% FBS and allowed to adhere overnight. On day 0 , both PAEC and PASMC media was changed to DMEM plus $2.5 \%$ FBS. PAEC containing Transwells were placed in the six-well plates with the PASMC. On day 4, PASMC were removed from the wells using $0.25 \%$ trypsin $/ 0.53 \mathrm{mmol} / \mathrm{l}$ EDTA digestion and counted using a hemocytometer. For all coculture experiments, comparisons were made of absolute number of PASMC present on day 4 . Two experimental setups were used as follows:

1. Control PASMC with either control or CDH PAEC

2. CDH PASMC with either control or CDH PAEC

\section{Western Blot Analysis}

PAEC from normal and $\mathrm{CDH}$ lambs were grown on $150 \mathrm{~mm}$ cloning plates with DMEM and 10\% FBS. When the cells reached 100\% confluence, cell lysates were collected, as described previously (38). Protein content of samples was determined using the BCA protein assay (Pierce Biotechnology, Rockford, IL), using bovine serum albumin as the standard. A $25 \mu \mathrm{g}$ protein sample was added to each lane and resolved by sodium dodecyl sulfate-polyacrylamide gel electrophoresis. Proteins from the gel were then transferred to a nitrocellulose membrane. ET-1 (Pierce Antibodies, Thermo Fisher Scientific, Rockford, IL) and $\beta$-actin (Sigma Aldrich) were detected as previously described (39) using appropriate controls and molecular weight as identified by the manufacturer for the protein of interest. Densitometry was performed using Image Lab (version 4.0.1; BioRad Laboratories, Hercules, CA). Changes in protein expression were analyzed after normalizing for $\beta$-actin expression. The same protocol was used to evaluate protein content of PASMC lysates. PASMC from control or the $\mathrm{CDH}$ animals were collected as described above. Following gel electrophoresis, ET-1 (Pierce Antibodies, Thermo Fisher Scientific), ET-A receptor (Abcam, Cambridge, MA), ET-B receptor (Enzo Life Sciences, Farmingdale, NY), and $\beta$-actin (Sigma Aldrich) were detected as previously described (38) using appropriate controls and molecular weight as identified by the manufacturer for the protein of interest.

\section{Measurement of ET-1 Protein in Supernatant}

PAEC and PASMC from control and CDH fetal sheep were grown to $90 \%$ confluence in $60 \mathrm{~mm}$ dishes, supernatant removed and spun down at $1,800 \mathrm{rpm}$, so as to remove floating debris and cells. ET-1 protein in the supernatant from control and CDH PAEC and PASMC was assayed using Endothelin-1 ELIS kit (Enzo Life Sciences (Farmingdale, NY) (Catalog number ADI-900-020A) per manufacturers recommendation. Briefly for PAECs, an equal volume $(100 \mu \mathrm{l})$ of assay buffer and protein standards and sample are added to each well in triplicate. After a $1 \mathrm{~h}$ incubation, the plate is washed five times with washing buffer, after which antibody is added to each well. The plate is incubated for $30 \mathrm{~min}$ and washed as above. Substrate solution is added to each well followed by a $30 \mathrm{~min}$ incubation after which stop solution is added and optical density read at $450 \mathrm{~nm}$. To account for background, a blank well is utilized and the background reading in the blank well subtracted from the samples. As ET-1 in the PASMC supernatant was below the minimum detection range (1-3 $\mathrm{pg} / \mathrm{ml}$ ), sample extraction was performed using sep-Pak $\mathrm{C}_{18}$ columns (Peninsula Laboratories, LLC \# Y-1000). Prior to loading an equal volume of $20 \%$ acetic acid was added to each sample to acidify the solution. Solid phase extraction was performed and samples lyophilized overnight using a centrifugal concentrator. Purified lyophilized samples were resuspended in assay buffer and ELISA was performed in triplicate as above, per manufacturers recommendation.

\section{PAEC Growth}

Fetal PAEC from normal and CDH lambs were plated on a six-well tissue culture plate at $1 \times 10^{6}$ cells/well in DMEM with $10 \% \mathrm{FBS}$ and allowed to adhere overnight. On day 0 , media was changed to DMEM with 2.5\% FBS (as this was the lowest concentration that supported growth) with or without the addition of SOD $(250 \mathrm{U} / \mathrm{ml})$ plus catalase $(26.3 \mathrm{U} / \mathrm{ml})$. Media was changed daily. On day 3 , cells were removed from the wells using $0.25 \%$ trypsin $/ 0.53 \mathrm{mmol} / \mathrm{l}$ EDTA digestion and counted using a hemocytometer. Absolute cell number on day 3 was compared to cell number at day 0 to determine the relative increase over time.

\section{Tube Formation Assay}

PAEC from normal and CDH lambs were plated on collagen coated wells to assess the ability of the cells to form capillary like structures, as previously described (36). $4 \times 10^{4}$ cells/well were initially plated in serum-free DMEM supplemented with and without SOD $(250 \mathrm{U} / \mathrm{ml})$ plus catalase $(26.3 \mathrm{U} / \mathrm{ml})$. Cells were incubated for $18 \mathrm{~h}$ in room air conditions. Counting of tubular structures was performed under $10 \times$ magnification from four different locations in each well.

\section{ROS Production in PAEC}

ROS production, including superoxide and hydrogen peroxide, were quantified utilizing the CellROX Deep Red reagent (Life Technologies, Thermo Fisher Scientific). CellROX is a fluorogenic probe, which is nonfluorescent in the reduced state and becomes fluorescent upon oxidation by ROS with absorption/emission maxima of $644 / 665 \mathrm{~nm} .1 \times 10^{5}$ PAEC from control and $\mathrm{CDH}$ sheep were plated in a 24-well tissue culture dish and allowed to adhere. Cells were cultured in DMEM with 10\% FBS for 48-72 h until 80-90\% confluence was reached. Cells were then washed two times with PBS after which $0.5 \mathrm{ml}$ phenol red and serum free media was added to each well and cells were incubated for $30 \mathrm{~min}$. Conditions requiring pretreatment were added prior to incubation. These include SOD $(250 \mathrm{U} / \mathrm{ml})$ plus catalase $(26.3 \mathrm{U} / \mathrm{ml})$ and ET- $1(25 \mathrm{ng} / \mathrm{ml})$. After the $30 \mathrm{~min}$ incubation, media was removed and replaced with $0.5 \mathrm{ml}$ of phenol red and serum free media with CellROX $(5 \mu \mathrm{mol} / \mathrm{l})$ plus indicated conditions and incubated for $45 \mathrm{~min}$. Following incubation, fluorescence was measured utilizing a fluorescent plate reader at absorption/emission maxima of $644 / 665 \mathrm{~nm}$ and results were corrected for cell number. In addition to those mentioned above (SOD plus catalase and ET-1), conditions used include hypoxanthine (HX) $(0.5 \mathrm{mmol} / \mathrm{l})$ plus xanthine oxidase $(\mathrm{XO})(8 \mathrm{mU} / \mathrm{ml})$ as a positive control and leads to a maximum fluorescent signal in both control and CDH PAEC (data not shown). Additionally, the assay was repeated utilizing PAEC from $\mathrm{CDH}$ sheep that were transfected with ET-1 siRNA (protocol described below).

\section{siRNA Transfection of PAEC}

ET-1 siRNA (h) (Santra Cruz Biotechnology, Santa Cruz, CA) was used for siRNA transfection as previously described (40). Briefly, in a six-well tissue culture plate, $1 \times 10^{6}$ PAEC were plated in $2 \mathrm{ml}$ DMEM with $10 \%$ FBS and allowed to adhere for $48 \mathrm{~h}$ at which time media was removed and transfection reagents (negative control, scramble siRNA and ET-1 siRNA) were added to duplicate wells for a final volume of 
$1 \mathrm{ml}$. ET-1 siRNA ( $4 \mu \mathrm{mol} / \mathrm{l})$, negative control, and scramble siRNA (Santra Cruz Biotechnology) were mixed with equal parts transfection reagent and added to the appropriate wells for $6 \mathrm{~h}$ at which time $1 \mathrm{ml}$ DMEM with 20\% FBS was added to each well without removal of the transfection reagent (Santra Cruz Biotechnology). After 18$24 \mathrm{~h}$, transfection media was replaced with DMEM with $10 \%$ FBS. After an additional $48 \mathrm{~h}$, cells were utilized for the experiments listed below. There was no further exposure to siRNA; transfected cells were handled and utilized in the same manner as comparison cells. Transfection efficiency was confirmed following isolation of cellular RNA with PCR, which revealed a 50\% decrease in total ET-1 protein content.

\section{Coculture Studies of PAEC With PASMC}

The initial coculture set up in which control PASMC were cocultured with either control or CDH PAEC was repeated with and without the addition of SOD $(250 \mathrm{U} / \mathrm{ml})$ plus catalase $(26.3 \mathrm{U} / \mathrm{ml})$. In this case, SOD and catalase were added to the DMEM with $2.5 \%$ FBS on day 0 , media were changed daily and PASMC cell counts were performed on day 4. And finally, the coculture set up was repeated using PAEC from both control and CDH lambs following transfection with ET-1 siRNA. These transfected endothelial cells were cocultured with nontransfected PASMC from a single control animal. This experimental set up was performed twice.

\section{Statistical Analysis}

Data are presented as means \pm SEM. Data from all animals in the control and $\mathrm{CDH}$ groups were combined for data analysis. An unpaired $t$-test or Mann-Whitney test was used where appropriate to compare parametric and non parametric data. Other statistical comparisons were made with one-way ANOVA. Post hoc analysis used Fisher's least significant difference test. (Prism 6 software; GraphPad Software, La Jolla, CA). $P$ values less than 0.05 were considered statistically significant.

\section{STATEMENT OF FINANCIAL SUPPORT}

This work was supported in part by grants from the U.S. National Institutes of Health RO1 HL085703 (S.H.A.), U01 HL102235 (S.H.A.), RO1 HL68702 (S.H.A.), RO1 HL086680 (E.N-.G.), and NHLBI K08HL102261 (J.G.); as well as the Entelligence Young Investigator Award (J.G.). The Actelion ENTELLIGENCE program is supported through an educational grant from Actelion Pharmaceuticals US, Inc.

Disclosure: The authors declare no conflict of interest.

\section{REFERENCES}

1. Downard CD, Jaksic T, Garza JJ, et al. Analysis of an improved survival rate for congenital diaphragmatic hernia. J Pediatr Surg 2003;38:729-32.

2. Taira Y, Yamataka T, Miyazaki E, Puri P. Comparison of the pulmonary vasculature in newborns and stillborns with congenital diaphragmatic hernia. Pediatr Surg Int 1998;14:30-5.

3. Dillon PW, Cilley RE, Mauger D, Zachary C, Meier A. The relationship of pulmonary artery pressure and survival in congenital diaphragmatic hernia. J Pediatr Surg 2004;39:307-12; discussion 307-12.

4. Reiss I, Schaible T, van den Hout L, et al.; CDH EURO Consortium. Standardized postnatal management of infants with congenital diaphragmatic hernia in Europe: the CDH EURO Consortium consensus. Neonatology 2010;98:354-64.

5. Skari H, Bjornland K, Haugen G, Egeland T, Emblem R. Congenital diaphragmatic hernia: a meta-analysis of mortality factors. J Pediatr Surg 2000;35:1187-97.

6. Suda K, Bigras JL, Bohn D, Hornberger LK, McCrindle BW. Echocardiographic predictors of outcome in newborns with congenital diaphragmatic hernia. Pediatrics 2000;105:1106-9.

7. Acker SN, Seedorf GJ, Abman SH, Nozik-Grayck E, Partrick DA, Gien J. Pulmonary artery endothelial cell dysfunction and decreased populations of highly proliferative endothelial cells in experimental congenital diaphragmatic hernia. Am J Physiol Lung Cell Mol Physiol 2013;305:L943-52.

8. Thébaud B, Petit T, De Lagausie P, Dall'Ava-Santucci J, Mercier JC, DinhXuan AT. Altered guanylyl-cyclase activity in vitro of pulmonary arteries from fetal lambs with congenital diaphragmatic hernia. Am J Respir Cell Mol Biol 2002;27:42-7.

9. Antonelli-Orlidge A, Saunders KB, Smith SR, D'Amore PA. An activated form of transforming growth factor beta is produced by cocultures of endothelial cells and pericytes. Proc Natl Acad Sci USA 1989;86:4544-8.

10. Asakawa $\mathrm{H}$, Kobayashi $\mathrm{T}$. The effect of coculture with human smooth muscle cells on the proliferation, the IL-1 beta secretion, the PDGF production and tube formation of human aortic endothelial cells. Cell Biochem Funct 1999;17:123-30.

11. Hellström M, Kalén M, Lindahl P, Abramsson A, Betsholtz C. Role of PDGF-B and PDGFR-beta in recruitment of vascular smooth muscle cells and pericytes during embryonic blood vessel formation in the mouse. Development 1999;126:3047-55.

12. Hirschi KK, Rohovsky SA, D’Amore PA. PDGF, TGF-beta, and heterotypic cell-cell interactions mediate endothelial cell-induced recruitment of $10 \mathrm{~T} 1 / 2$ cells and their differentiation to a smooth muscle fate. J Cell Biol 1998;141:805-14.

13. Lindahl P, Johansson BR, Levéen P, Betsholtz C. Pericyte loss and microaneurysm formation in PDGF-B-deficient mice. Science 1997;277:242-5.

14. Huang B, Che D, Zhang W. Effects of the medium conditioned by endothelial cells under hypoxic condition on the phenotype of porcine pulmonary arterial smooth muscle cells. J Tongji Med Univ 1997;17:209-12.

15. Wedgwood S, Black SM. Endothelin-1 decreases endothelial NOS expression and activity through ETA receptor-mediated generation of hydrogen peroxide. Am J Physiol Lung Cell Mol Physiol 2005;288:L480-7.

16. Wedgwood S, Dettman RW, Black SM. ET-1 stimulates pulmonary arterial smooth muscle cell proliferation via induction of reactive oxygen species. Am J Physiol Lung Cell Mol Physiol 2001;281:L1058-67.

17. Budhiraja R, Tuder RM, Hassoun PM. Endothelial dysfunction in pulmonary hypertension. Circulation 2004;109:159-65.

18. Ivy DD, Le Cras TD, Horan MP, Abman SH. Increased lung preproET-1 and decreased ETB-receptor gene expression in fetal pulmonary hypertension. Am J Physiol 1998;274(4 Pt 1):L535-41.

19. Hassoun PM, Thappa V, Landman MJ, Fanburg BL. Endothelin 1: mitogenic activity on pulmonary artery smooth muscle cells and release from hypoxic endothelial cells. Proc Soc Exp Biol Med 1992;199:165-70.

20. Keller RL, Tacy TA, Hendricks-Munoz K, et al. Congenital diaphragmatic hernia: endothelin-1, pulmonary hypertension, and disease severity. Am J Respir Crit Care Med 2010;182:555-61.

21. Kobayashi H, Puri P. Plasma endothelin levels in congenital diaphragmatic hernia. J Pediatr Surg 1994;29:1258-61.

22. Rosenberg AA, Kennaugh J, Koppenhafer SL, Loomis M, Chatfield BA, Abman SH. Elevated immunoreactive endothelin-1 levels in newborn infants with persistent pulmonary hypertension. J Pediatr 1993;123: $109-14$.

23. Okazaki T, Sharma HS, McCune SK, Tibboel D. Pulmonary vascular balance in congenital diaphragmatic hernia: enhanced endothelin-1 gene expression as a possible cause of pulmonary vasoconstriction. J Pediatr Surg 1998;33:81-4.

24. Lin H, Wang Y, Xiong Z, Tang Y, Liu W. Effect of antenatal tetrandrine administration on endothelin-1 and epidermal growth factor levels in the lungs of rats with experimental diaphragmatic hernia. J Pediatr Surg 2007;42:1644-51.

25. Steinhorn RH, Albert G, Swartz DD, Russell JA, Levine CR, Davis JM. Recombinant human superoxide dismutase enhances the effect of inhaled nitric oxide in persistent pulmonary hypertension. Am J Respir Crit Care Med 2001;164:834-9.

26. Ahmed MN, Suliman HB, Folz RJ, et al. Extracellular superoxide dismutase protects lung development in hyperoxia-exposed newborn mice. Am J Respir Crit Care Med 2003;167:400-5.

27. Nozik-Grayck E, Suliman HB, Majka S, et al. Lung EC-SOD overexpression attenuates hypoxic induction of Egr-1 and chronic hypoxic pulmonary vascular remodeling. Am J Physiol Lung Cell Mol Physiol 2008;295:L422-30.

28. Gien J, Tseng N, Seedorf G, Roe G, Abman SH. Peroxisome proliferator activated receptor-?-Rho-kinase interactions contribute to vascular remodeling after chronic intrauterine pulmonary hypertension. Am J Physiol Lung Cell Mol Physiol 2014;306:L299-308. 
29. Dewachter L, Adnot S, Guignabert C, et al. Bone morphogenetic protein signalling in heritable versus idiopathic pulmonary hypertension. Eur Respir J 2009;34:1100-10.

30. Falcetti E, Hall SM, Phillips PG, et al. Smooth muscle proliferation and role of the prostacyclin (IP) receptor in idiopathic pulmonary arterial hypertension. Am J Respir Crit Care Med 2010;182: 1161-70.

31. Janakidevi K, Tiruppathi C, Del Vecchio PJ, Pinheiro JM, Malik AB. Growth characteristics of pulmonary artery smooth muscle cells from fawn-hooded rats. Am J Physiol 1995;268(3 Pt 1):L465-70.

32. Kavanagh M, Seaborn T, Crochetière J, et al. Modulating effect of a selective endothelin A receptor antagonist on pulmonary endothelin system protein expression in experimental diaphragmatic hernia. J Pediatr Surg 2005;40:1382-9.

33. Brennan LA, Steinhorn RH, Wedgwood S, et al. Increased superoxide generation is associated with pulmonary hypertension in fetal lambs: a role for NADPH oxidase. Circ Res 2003;92:683-91.

34. Brown MR, Miller FJ Jr, Li WG, et al. Overexpression of human catalase inhibits proliferation and promotes apoptosis in vascular smooth muscle cells. Circ Res 1999;85:524-33.
35. Tsai JC, Jain M, Hsieh CM, et al. Induction of apoptosis by pyrrolidinedithiocarbamate and $\mathrm{N}$-acetylcysteine in vascular smooth muscle cells. J Biol Chem 1996;271:3667-70.

36. Gien J, Seedorf GJ, Balasubramaniam V, Markham N, Abman SH. Intrauterine pulmonary hypertension impairs angiogenesis in vitro: role of vascular endothelial growth factor nitric oxide signaling. Am J Respir Crit Care Med 2007;176:1146-53.

37. Konduri GG, Ou J, Shi Y, Pritchard KA Jr. Decreased association of HSP90 impairs endothelial nitric oxide synthase in fetal lambs with persistent pulmonary hypertension. Am J Physiol Heart Circ Physiol 2003;285:H204-11.

38. Balasubramaniam V, Maxey AM, Fouty BW, Abman SH. Nitric oxide augments fetal pulmonary artery endothelial cell angiogenesis in vitro. Am J Physiol Lung Cell Mol Physiol 2006;290:L1111-6.

39. Balasubramaniam V, Maxey AM, Morgan DB, Markham NE, Abman SH. Inhaled $\mathrm{NO}$ restores lung structure in eNOS-deficient mice recovering from neonatal hypoxia. Am J Physiol Lung Cell Mol Physiol 2006;291:L119-27.

40. Wolf D, Tseng N, Seedorf G, Roe G, Abman SH, Gien J. Endothelin-1 decreases endothelial PPAR? signaling and impairs angiogenesis after chronic intrauterine pulmonary hypertension. Am J Physiol Lung Cell Mol Physiol 2014;306:L361-71. 